\title{
THE WALLMAN COMPACTIFICATION IS AN EPIREFLECTION
}

\author{
DOUGLAS HARRIS
}

\begin{abstract}
It is shown that a map having an extension to a closed map between the Wallman compactifications of its domain and range has a unique such extension. A consequence is that the collection of such maps forms the morphisms of a category on which the Wallman compactification is an epireflection, answering a question raised by Herrlich.
\end{abstract}

We establish the existence of a category $\mathcal{C}$ of spaces and maps on which the Wallman compactification is an epireflection functor. This answers a question raised by Herrlich in [1] as to whether such a category exists.

A space is a $T_{1}$ topological space and a map is a continuous function. For categorical terminology see [1].

The class of objects of $\mathrm{C}$ will be the class of spaces, and the morphisms will be the class of maps $f: X \rightarrow Y$ such that there is a closed map $g: w X \rightarrow w Y$ with $g w_{X}=w_{Y} f$, where $w_{X}, w_{Y}$ are the inclusions of $X, Y$ into their Wallman compactifications $w X, w Y$. Any such closed map $g$ is called a w-extension of $f$.

It is clear that for each $X \in \mathrm{C}$ the maps $1_{X}$ and $w_{X}$ are morphisms, since $1_{w X}$ is a $w$-extension of these maps. Also trivially if $f$ is a morphism and $g$ is a $w$-extension of $f$ then $g$ is a morphism (being its own $w$-extension). Finally since the composition of closed maps is a closed map it follows that the composition of morphisms is a morphism.

To show that the Wallman compactification is an epireflection on this category we need only show that each morphism has a unique $w$-extension. To do this we examine the construction of the Wallman compactification of $X$ as given for example in [2].

The points of the Wallman compactification are the maximal closed filters $[p]$ on $X$; we write $[p]$ for the filter and $p$ for the point of $w X$. For each $p \in w X$ there is the open filter $\langle p\rangle$ consisting of the open subsets of $Y$ which contain a member of $[p]$. The neighborhood filter of $p \in w X$ is

Received by the editors February 10, 1971.

AMS 1970 subject classifications. Primary 54D35.

Key words and phrases. Wallman compactification, closed map, epireflection, maximal closed filter. 
generated by the sets $V^{*}=\{q \in w X: V \in\langle q\rangle\}$ taken over all $V \in\langle p\rangle$. Since for each $x \in X$ the principal filter $[x]$ is maximal closed we have the function $w_{X}$, and it is an embedding of $X$ into $w X$.

LemMa. Iff $: X \rightarrow Y$ is a morphism and $g: w X \rightarrow w Y$ is a w-extension of $f$ then for any open $U \subset Y$ we have $U \in\langle g(p)\rangle$ if and only if there is $C \in[p]$ with $\mathrm{cl}_{Y} f[C] \subset U$.

Proof. Suppose $C \in[p]$. Then $p \in \mathrm{cl}_{w X} w_{X}[C]$ and so

$$
g(p) \in g\left[\mathrm{cl}_{w X} w_{X}[C]\right] \subset \mathrm{cl}_{w Y} g w_{X}[C]=\mathrm{cl}_{w Y} w_{Y} f[C] ;
$$

therefore $\operatorname{cl}_{Y} f[C] \in[g(p)]$. Thus if $\operatorname{cl}_{Y} f[C] \subset U$ for some $C \in[p]$ and open $U \subset Y$ then $B \subset U$ for some $B \in[g(p)]$, and thus $U \in\langle g(p)\rangle$.

Conversely suppose $B \subset U$ for some $B \in[g(p)]$ and open $U \subset Y$. Then $g^{\leftarrow}\left[U^{*}\right]$ is a neighborhood of $p$, so there is $W \in\langle p\rangle$ with $W^{*} \subset$ $g^{\leftarrow}\left[U^{*}\right]$. Since $W \in\langle p\rangle$ there is $C \in[p]$ with $C \subset W$. Now $\mathrm{cl}_{w X} w_{X}[C]=$ $C^{*} \subset W^{*}$, and since $g$ is closed we have $\operatorname{cl}_{w Y} w_{Y} f[C]=\operatorname{cl}_{w Y} g w_{X}[C]=$ $\operatorname{cl}_{{ }_{w} Y} g\left[\mathrm{cl}_{w X} w_{X}[C]\right]=g\left[C^{*}\right] \subset g\left[W^{*}\right] \subset U^{*} ;$ therefore $\operatorname{cl}_{Y} f[C] \subset U$. Thus if $U \in\langle g(p)\rangle$ there is $C \in[p]$ with $\operatorname{cl}_{Y} f[C] \subset U$.

In accordance with the Lemma the filter $\langle g(p)\rangle$ and hence the point $g(p)$ is entirely determined by the map $f$, hence the following holds:

COROLlary 1. Each morphism has a unique w-extension.

Now suppose $g$ and $h$ are morphisms with $g w_{X}=h w_{X}$. Then $g$ and $h$ have $w$-extensions $m$ and $n$. Since $n w_{X}=w_{Y} g w_{X}=w_{Y} h w_{X}=m w_{X}$ then by the preceding corollary $n=m$ and therefore $w_{Y} h=n=m=$ $w_{Y} g$, from which we find $h=g$. We therefore find the further result:

COROLlaRY 2. Each $w_{X}$ is an epimorphism.

Summing up the preceding results, and making the trivial observation that each morphism with compact domain and range is a closed map we find:

THEOREM. The category of closed maps and compact spaces is an epireflective subcategory of the category $\mathrm{C}$, and the epireflection of a space is its Wallman compactification.

It is known that every closed onto map has a closed onto $w$-extension; see Ponomarev [3] for this result, first shown by Arhangel'skiir. Thus each closed onto map is a morphism. Another class of morphisms is the class of maps with compact Hausdorff range; as is well known (and readily established using extension by regularity) each such map has an extension to the Wallman compactification of its domain, and the extension must be closed. 


\section{REFERENCES}

1. H. Herrlich, On the concept of reflections in general topology, Proc. First Internat. Sympos. on Extension Theory of Topological Structures, VEB Deutscher Verlag Wissenschaften, Berlin, 1969.

2. J. L. Kelley, General topology, Van Nostrand, Princeton, N.J., 1955. MR 16, 1136.

3. V. I. Ponomarev, On the extension of multivalued mappings of topological spaces to their compactifications, Mat. Sb. 52 (94) (1960), 847-862; English transl., Amer. Math. Soc. Transl. (2) 38 (1964), 141-158. MR 22 \#12513.

Department of Mathematics, Marquette University, Milwaukee, Wisconsin 53233 